\title{
Using Technology to Reshape Clinical Care and Research in Parkinson's Disease
}

\author{
Anat Mirelman ${ }^{\mathrm{a}, \mathrm{b}}$, E. Ray Dorsey ${ }^{\mathrm{c}}$, Patrik Brundin ${ }^{\mathrm{d}}$ and Bastiaan R. Bloem ${ }^{\mathrm{e}, \mathrm{f}, *}$ \\ ${ }^{a}$ Laboratory for Early Markers Of Neurodegeneration, Center for The Study of Movement, Cognition and \\ Mobility, Neurological Institute, Tel Aviv Sourasky Medical Center, Tel Aviv Israel \\ ${ }^{\mathrm{b}}$ Sackler Faculty of Medicine, and Sagol School of Neuroscience, Tel Aviv University, Israel \\ ${ }^{\mathrm{c}}$ Department of Neurology, Centre for Health + Technology, University of Rochester Medical Centre, Rochester, \\ New York, USA \\ ${ }^{\mathrm{d}}$ Laboratory of Translational Parkinson's Disease Research, Center for Neurodegenerative Science, Van Andel \\ Research Institute, Grand Rapids, MI, USA \\ ${ }^{\mathrm{e}}$ Department of Neurology, Radboud University Nijmegen Medical Center, Nijmegen, The Netherlands \\ ${ }_{\mathrm{f}}^{\mathrm{f}}$ Donders Institute for Brain, Cognition and Behavior, Radboud University, Nijmegen, The Netherlands
}

Pre-press 16 February 2021

Keywords: Neurological examination, expertise, clinical skills, Parkinson's disease, movement disorders

The digital revolution has impacted every facet of society, and medical care and research are no exceptions. A variety of digital technologies, designed to either capture clinically relevant health-related data or to facilitate communication between healthcare professionals and patients, have emerged in the past two decades. Digital health technology is an umbrella term that spans a very diverse range of appliances, including body-fixed wearable sensors, non-contactable domestic sensors, apps for the smartphone, and videoconferencing or other telemedicine systems that allow for direct remote interaction with patients.

These digital tools offer several promises, one of which is the ability to objectively quantify a variety of signs and symptoms in the clinic and at home. In Parkinson's disease, continuous assessment during the day or over even longer periods of time in

\footnotetext{
*Correspondence to: Prof. Bastiaan R. Bloem, MD, PhD, FRCPE, Department of Neurology (935), Radboud University Nijmegen Medical Center, PO Box 9101, 6500 HB Nijmegen, The Netherlands. Tel.: +31 243615 202; E-mail: bas.bloem@ radboudumc.nl.
}

the patients' own natural environment can be especially useful to help assess variations in the severity and nature of symptoms, and to avoid wrongful representation of symptoms due to "white coat syndrome" or negative stress during assessment. Furthermore, changes in therapeutic regimens typically take weeks before they fully impact symptoms and can be subject to observer bias. The use of digital monitoring over several months can circumvent these problems.

During the past decade, many research groups have explored the validity of such technology-based objective measures, resulting in numerous papers from different disciplines with mixed results [1-3]. For example, mobility measures evaluated in the unstructured home-environment differ extensively from those measured in a much more structured clinical setting. These differences may stem from the environment, but also from properties of the movement itself, as mobility in a free-living environment is typically self-initiated, embedded in a rich behavioural context, and goal-directed, unlike that in a controlled experimental testing situation [4]. Such discrepancies challenge not the validity of the measurements, but 
rather questions what is really examined in each of these settings; the clinic versus the home [5, 6].

Nonetheless, the field has seen an accumulating body of evidence to support the feasibility, utility and benefits of various digital technology approaches $[1,7,8]$. Despite this fundamental progress, the use of digital technology in clinical practice has long remained scarce. It took a contagion to make patients, physicians and insurers pay better attention. Specifically, the limits on our ability to travel imposed by the ongoing coronavirus pandemic (COVID-19) led to a dramatic increase in the use of digital platforms in a matter of a few months. This change was unprecedented and has been identified as one of the few silver linings of the pandemic. The increase in use of digital platforms is transforming neurological care at a rapid pace, catalysing the use of digital technology to maintain continuity of care, and also that of clinical trials [7-9]. The need for social isolation to mitigate the risk of becoming infected has fostered the expansion of telemedicine programs in many clinics around the world [10-12]. Using wearable sensors, clinicians are now still able to monitor their patients' motor symptoms while they are at home [13] and gain insight into behavioural changes and the implications of social distancing on patients' non-motor symptoms $[14,15]$. Wearable technology also enabled the monitoring of multidimensional physiological measures for the timely detection of COVID-19 in patients, [16] showing the feasibility of use in yet another area of care. While these various developments are encouraging, many vexing questions remain with respect to accessibility, acceptance and regulation of digital health technologies, as well as their actual seamless incorporation into daily clinical practice. The COVID-19 pandemic has also served to emphasise some of the shortcomings of telemedicine or monitoring approaches, such as the undiminished need for in-person visits, especially for new patients [17]. Such in-person visits also remain essential for building up an intimate relationship between the team of healthcare providers and the patient and his or her near ones. These shortcomings raise yet another question as to what extent digital health technologies will survive when our lives get back to a new normal after this pandemic has resolved.

In clinical research, digital technologies are poised to transform the current practice and enhance our understanding of Parkinson's disease by extending the reach and expanding the scope of our investigations [18]. Current assessments of Parkinson's disease are largely limited. For example, sleep assessments are limited to episodic polysomnograms performed in artificial settings at an arbitrary time. Assessing sleep can now be done in the home, passively, and frequently. Such measures will vastly expand our knowledge of sleep disorders in those with clinically manifest Parkinson's and presumably also in individuals with a prodromal presentation.

These new tools may also shine a light on features of the disease that have largely been invisible to us, such as the appraisal of social function (e.g., how much time an individual spends at home or with others), expanded assessments of voice, quantification of tremor, assessment of falls, dyskinesia or freezing of gait and objective measurement of the response to treatment. This work could lead to a better understanding of the disease, its temporal pattern, variability, and impact on individuals bearing its burden. In addition, much needed new, objective digital endpoints may emerge that can be assessed in the real world.

Finally, these technological applications have the potential to re-shape how we conduct clinical research as decentralized trials will expand and even more assessments will transfer from the clinic to the home and mobile devices [18]. Digital measurement of the disease will lay the foundation for digital treatments. Some of these digital therapeutics may be expansions of existing therapies (e.g., deep brain stimulation), while others may be entirely novel (e.g., cognitive behavioural therapy for anxiety or music for gait impairment). For some therapies, remote digital therapeutics can offer an attractive alternative for intensive interventions such as speech and language therapy, which typically requires frequent visits to clinics; research is now ongoing to study the merits of completely remote speech therapy.

In this special issue of the Journal of Parkinson's Disease, we provide glimpses into that future of digital health. This issue on the state of digital health in Parkinson's disease includes a series of succinct reviews that cover many practical and relevant aspects, such as digital monitoring, telemedicine, digital therapeutics, virtual clinical trials and digital progression biomarkers for clinical trials. All reviews offer both a view of the current state-of-theart and informed perspectives on future directions. We can say with certainty that the digital revolution has definitely started for Parkinson's disease care and research. We can almost be equally certain that this is only the beginning and that the future of digital medicine will likely benefit both patients and health care in ways that currently are difficult to predict. 


\section{ACKNOWLEDGMENTS}

The Radboudumc Center of Expertise for Parkinson \& Movement Disorders is supported by a center of excellence grant of the Parkinson's Foundation.

\section{DISCLOSURES}

None of the authors has any relevant disclosures to make.

\section{REFERENCES}

[1] Arroyo-Gallego T, Ledesma-Carbayo MJ, Butterworth I, et al. (2018) Detecting Motor Impairment in Early Parkinson's Disease via Natural Typing Interaction With Keyboards: Validation of the neuroQWERTY Approach in an Uncontrolled At-Home Setting. J Med Internet Res $\mathbf{2 0}$ e89.

[2] Hillel I, Gazit E, Nieuwboer A, et al. (2019) Is every-day walking in older adults more analogous to dual-task walking or to usual walking? Elucidating the gaps between gait performance in the lab and during 24/7 monitoring. Eur Rev Aging Phys Act 16, 6.

[3] Takayanagi N, Sudo M, Yamashiro Y, et al. (2019) Relationship between Daily and In-laboratory Gait Speed among Healthy Community-dwelling Older Adults. Sci Rep 9, 3496.

[4] Warmerdam E, Hausdorff JM, Atrsaei A, et al. (2020) Long-term unsupervised mobility assessment in movement disorders. Lancet Neurol 19, 462-470.

[5] Galperin I, Hillel I, Del DS, et al. (2019) Associations between daily-living physical activity and laboratory-based assessments of motor severity in patients with falls and Parkinson's disease. Parkinsonism Relat Disord 62, 85-90.

[6] Maetzler W, Rochester L, Bhidayasiri R, et al. Modernizing Daily Function Assessment in Parkinson's Disease Using Capacity, Perception, and Performance Measures. Mov Disord. 2020.
[7] Bloem BR, Dorsey ER, Okun MS (2020) The Coronavirus Disease 2019 Crisis as Catalyst for Telemedicine for Chronic Neurological Disorders. JAMA Neurol 77, 927-928.

[8] Dorsey ER, Okun MS, Bloem BR (2020) Care, Convenience, Comfort, Confidentiality, and Contagion: The $5 \mathrm{C}$ 's that Will Shape the Future of Telemedicine. J Parkinsons Dis 10, 893-897.

[9] Miele G, Straccia G, Moccia M, et al. (2020) Telemedicine in Parkinson's Disease: How to Ensure Patient Needs and Continuity of Care at the Time of COVID-19 Pandemic. Telemed J E Health 26, 1533-1536.

[10] Cohen E, Cohen MI (2021) COVID-19 will forever change the landscape of telemedicine. Curr Opin Cardiol 36, 110-115.

[11] Dorsey ER, Bloem BR, Okun MS (2020) A New Day: The Role of Telemedicine in Reshaping Care for Persons With Movement Disorders. Mov Disord 35, 1897-1902.

[12] Mulroy E, Menozzi E, Lees AJ, et al. (2020) Telemedicine in Movement Disorders: Lecons du COVID-19. Mov Disord 35, 1893-1896.

[13] Motolese F, Magliozzi A, Puttini F, et al. (2020) Parkinson's Disease Remote Patient Monitoring During the COVID-19 Lockdown. Front Neurol 11, 567413.

[14] Kyritsis K, Fagerberg P, Ioakimidis I, et al. (2021) Assessment of real life eating difficulties in Parkinson's disease patients by measuring plate to mouth movement elongation with inertial sensors. Sci Rep 11, 1632.

[15] Sun S, Folarin AA, Ranjan Y, et al. (2020) Using Smartphones and Wearable Devices to Monitor Behavioral Changes During COVID-19. J Med Internet Res 22, e19992.

[16] Al-Emran M, Ehrenfeld JM (2021) Breaking out of the Box: Wearable Technology Applications for Detecting the Spread of COVID-19. J Med Syst 45, 20.

[17] Mulroy E, Menozzi E, Lees AJ, et al. (2020) Reply to: "A New Day: The Role of Telemedicine in Reshaping Care for Persons With Movement Disorders". Mov Disord 35, 1903-1904.

[18] Dorsey ER, Kluger B, Lipset CH (2020) The New Normal in Clinical Trials: Decentralized Studies. Ann Neurol 88, 863-866. 